\title{
Phosphorylation of Neurofilament Proteins in Intact Neurons: Demonstration of Phosphorylation in Cell Bodies and Axons
}

\author{
Mark M. Black' and Virginia M.-Y. Lee ${ }^{2}$ \\ ${ }^{1}$ Department of Anatomy, Temple University School of Medicine, and ${ }^{2}$ Division of Neuropathology, Department of \\ Pathology and Laboratory Medicine, University of Pennsylvania Medical School Philadelphia, Pennsylvania
}

The principal subunits of neurofilaments (NFs) of immature cultured sympathetic neurons have apparent $M$, of 68,000 and 145,000; a 200,000 $M$, subunit is also present, but at comparatively low levels. These subunits are referred to as the low (NFL), middle (NFM), and high (NFH) $M$, subunits, respectively. We studied the phosphorylation of NFL and NFM in these neurons in order to characterize the NFL and NFM isoforms generated by this important posttranslational modification.

NFL resolved into a single spot in 2-dimensional gels, although 2 spots were occasionally observed. NFM typically resolved into 3 variants, termed NFM a, b, and c, in order of increasing mobility, but as many as 6 variants were detected in some gels.

NFL and, to a much greater degree, NFM became labeled following incubation of intact neurons with ${ }^{32} \mathrm{P}_{-} \mathrm{PO}_{4}$. Although all 3 major NFM variants became labeled, NFM a was the most heavily labeled, followed by NFM b, and then NFM $c$. Two observations suggest that the generation of these 3 NFM variants is due to their phosphorylation. First, treatment of NFs with phosphatase prior to analysis reduced NFM to a single spot or band that comigrated with NFM c; NFM a and $b$ were completely eliminated. However, NFM $c$ was not fully dephosphorylated because it still reacted with a monoclonal antibody (mAb) specific for a phosphate-dependent epitope on NFM. Second, NFM was recognized by 4 mAbs to distinctly different phosphorylated epitopes of NFM, which suggested that at least 4 distinct sites on NFM can be phosphorylated in cultured neurons.

Explant cultures were used to study the phosphorylation of NFL and NFM in cell bodies and axons. In these cultures, a central cell body mass (CBM) $0.5 \mathrm{~mm}$ in diameter contains all of the cell bodies, while peripheral to the CBM is a halo of pure axons that extends for 4-6 $\mathrm{mm}$. These cultures were incubated with ${ }^{32} \mathrm{P}_{-} \mathrm{PO}_{4}$ and $\mathrm{CBM}$ and axon regions were analyzed separately. NFL became phosphorylated to a greater extent in the CBM than in axons. NFM also became labeled in the CBM and axons, although the relative labeling of NFM a, b, and $c$ in these regions differed considerably from each other and also from the pattern observed in whole

Received Sept. 21, 1987; revised Dec. 28, 1987; accepted Jan. 11, 1987.

This work is supported by grants NS18616 and NS23530. M.M.B. is a recipient of a Research Career Development Award. Thanks are expressed to Dr. R. H. K. Liem for providing sequence information on rat NFM prior to publication.

Correspondence should be addressed to Dr. Mark M. Black, Department of Anatomy, Temple University School of Medicine, 3420 N. Broad Street, Philadelphia, PA 19140.

Copyright (C) 1988 Society for Neuroscience $0270-6474 / 88 / 093296-10 \$ 02.00 / 0$ neurons (cell bodies plus neurites, see above). Specifically, NFM c was the most heavily labeled variant generated in the CBM, followed by NFM $b$, and then NFM a, whereas in axons, NFM c was absent and NFM a was much more heavily labeled than NFM b.

These findings are discussed in terms of specific phosphorylation events that occur in cell bodies and/or axons, and the possibility that NFM and NFL undergo cyclic phosphorylation and dephosphorylation locally within axons.

Neurofilaments (NFs) are prominent components of the neuronal cytoskeleton that are particularly conspicuous in axons and large dendrites (Peters et al., 1976). In mammalian nervous systems, NFs are composed of a triplet of proteins that have $M_{\text {r }}$ of $68,000,145,000$, and 200,000 on SDS gels (Hoffiman and Lasek, 1975; Liem et al., 1978; Schlaepfer and Freeman, 1978) and are referred to as the low-, middle-, and high-molecularweight neurofilament subunits, or NFL, NFM, and NFH, respectively. All 3 neurofilament proteins (NFP) can be phosphorylated in vitro or in vivo (Julien and Mushynski, 1982). NFL is relatively poorly phosphorylated $\left(1 \mathrm{~mol}\right.$ of $\mathrm{PO}_{4} / \mathrm{mol}$ of NFL), while NFM and NFH are extensively phosphorylated (6 or more mol of $\mathrm{PO}_{4} / \mathrm{mol}$ of NFM and 20 or more $\mathrm{mol} \mathrm{PO}_{4} / \mathrm{mol}$ of NFH) (Jones and Williams, 1982; Julien and Mushynski, 1983; Wong et al., 1984; Carden et al., 1985; Ksiezak-Reding and Yen, 1987). Recent structural analyses of NFP have shown that NFM and NFH have substantial regions that project away from the NF core (Geisler et al., 1983) and that phosphorylation of these proteins occurs only on their projection domains (Julien and Mushynski, 1983; Carden et al., 1985). Thus, phosphorylation may regulate the volume occupied by NF by altering the conformation of the projection domains of NFM and NFH.

To better understand the functional significance of NFP phosphorylation, we have focused on defining exactly where it occurs within intact neurons. Immunostaining analyses from several laboratories (Sternberger and Sternberger, 1983; Bennett and DiLullo, 1985; Lee et al., 1986, 1987; Peng et al., 1986; Oblinger, 1987) have provided indirect evidence that NFP phosphorylation occurs in the proximal $(\sim 100 \mu \mathrm{m})$ region of the axon. The extent to which NFP phosphorylation occurs elsewhere in the neuron is largely unknown, although some indirect evidence for phosphorylation occurring in neuronal perikarya and distally in axons has been presented (Carden et al., 1987; Lee et al., 1987; Nixon et al., 1987).

Recently, we have prepared and characterized monoclonal antibodies (mAbs) that recognized epitopes on the core or peripheral domain of NFP, as well as mAbs that can distinguish different states of NFM and NFH phosphorylation in rat (Lee 
et al., 1987). Using a combination of immunochemical and immunocytochemical methods, we were able to identify mAbs that can distinguish 3 different phosphorylation states of NFM. These $\mathrm{mAbs}$ are referred to as $\mathrm{P}[+], \mathrm{P}[++]$, and $\mathrm{P}[+++]$ depending on which phosphorylation state they recognize. As reported in Lee et al. (1987) and in more detail here, NFM can be resolved into 3 major variants by PAGE that are designated herc as NFM a, b, and $c$ in order of increasing electrophoretic mobility. The mAbs of the $\mathrm{P}[+]$ type recognize phosphorylation-dependent epitopes on NFM a, b, and $c ; P[++]$ mAbs recognize both NFM a and $b$; and $\mathrm{P}[+++] \mathrm{mAbs}$ react almost exclusively with NFM a. We have used these mAbs in combination with a culture system that permits the physical separation of peripherally located axons from cell bodies and dendrites (Peng et al., 1986) to examine NFP phosphorylation in different regions of the neuron. Our results show that NFP are initially phosphorylated in the cell body compartment and that this process continues in proximal as well as distal regions of the axonal compartment.

\section{Materials and Methods}

Cell culture and metabolic labeling. Dissociated and explant cultures of rat sympathetic neurons were prepared and maintained as described previously (Lee, 1985; Peng et al., 1985, 1986). Dissociated cultures were typically used after 1-2 weeks in vitro. Explant cultures were used after 3 weeks in culture. Explant cultures consist of a centrally situated cell body mass (CBM), which is $\sim 0.5 \mathrm{~mm}$ in diameter and contains all of the neuron cell bodies, their dendrites and the proximal portion $(\sim 100$ $\mu \mathrm{m}$ ) of their axons; surrounding the CBM is a cell body-free halo of axons, which extends for 4-6 mm in all directions (Peng et al., 1986).

Dissociated cultures were labeled with $100-200 \mu \mathrm{Ci} / \mathrm{ml}{ }^{35} \mathrm{~S}$-methionine (Amersham) either continuously for $3 \mathrm{~d}$ in complete medium, or were pulse-labeled for $10-15 \mathrm{~min}$ with $1 \mathrm{mCi} / \mathrm{ml}$ in methionine-free medium. In some experiments, pulse-labeled cultures were chased for varying times in unlabeled medium containing 10 times the normal methionine. In a few experiments, cultures were incubated with ${ }^{3} \mathrm{H}$ acetate (ICN) as described in Black and Keyser (1987). To label proteins with phosphate, dissociated and intact explant cultures were incubated with ${ }^{32} \mathrm{P}_{-} \mathrm{PO}_{4}$ (ICN or NEN), $0.1-1 \mathrm{mCi} / \mathrm{ml}$, for $1-2 \mathrm{hr}$ in phosphatefree HEPES-buffered medium. At the end of the labeling periods, cultures were rinsed twice with Tris-buffered saline $(50 \mathrm{~mm}$ Tris- $\mathrm{HCl}, \mathrm{pH}$ $7.5,150 \mathrm{~mm} \mathrm{NaCl}$ ). Dissociated cultures were either dissolved directly in SDS-containing buffers or extracted with Triton X-100 to prepare cytoskeletons prior to analysis of NFPs (see below). Explant cultures were separated into CBMs and axonal halos as described in Peng et al. (1986). These were then dissolved directly in $1 \%$ SDS containing protease inhibitors, $5 \mathrm{~mm} \mathrm{NaF}$, and $10 \mathrm{~mm}$ each of EDTA and EGTA prior to analysis. In some experiments, cytoskeletons were prepared from the axons (see below). In other experiments, explant cultures were separated into CBM and axons prior to labeling. In this case, the CBM were placed in $1 \mathrm{ml}$ of phosphate-free medium in an eppendorf tube, while the axons, which remained attached to the original culture dish, were overlayed with phosphate-free medium. The samples were incubated with ${ }^{32} \mathrm{P}$ $\mathrm{PO}_{4}$ and processed as described below.

Immunological analyses of neurofilament proteins. Whole-cell SDS extracts or cytoskeletons were prepared from labeled or unlabeled cultures as described in Black et al. (1986) and assayed for NFPs by immunoprecipitation or immunoblotting. Immunoblotting and the detection of bound antibody using the PAP method was as described previously (Black and Keyser, 1987; Lee et al., 1987). Immunoprecipitation was modified slightly from Black and Keyser (1987). Briefly, whole cultures or cytoskeletons from labeled cultures were dissolved in $1 \%$ SDS containing protease inhibitors, $5 \mathrm{~mm} \mathrm{NaF}$, and $10 \mathrm{~mm}$ each of EDTA and EGTA, and the resulting suspensions were incubated in a boiling water bath for $5 \mathrm{~min}$ and then clarified by centrifugation $(12,000 \times g$ for 10 $\min$ at $\left.4^{\circ} \mathrm{C}\right)$. The supernate was diluted with 5 or 10 vol of Tris-buffered saline (50 mM Tris- $\mathrm{NCl}, \mathrm{pH} 7.6,200 \mathrm{mM} \mathrm{NaCl}$ ) containing $1 \%(\mathrm{wt} / \mathrm{vol})$ NP-40 and then divided into appropriate numbers of equal-sized aliquots. The aliquots were incubated with or without antibody overnight at $4^{\circ} \mathrm{C}$, and then antibody-antigen complexes were harvested by incu-

\begin{tabular}{lll}
\hline Table 1. & Specificity of monoclonal antibodies & \\
mAbs & Subunit specificities & Epitope specificities \\
\hline RMS21 & NFL & P[ind-core $]$ \\
RMO26 & NFM & P[ind-core] \\
RMO108 & NFM & P $[+]$ \\
RMO55 & NFM & $\mathrm{P}[++]$ \\
RMO45 & NFM & $\mathrm{P}[+++]$ \\
SMI 31 & NFM/NFH & $\mathrm{P}[++]$
\end{tabular}

The subunit specificity and the location and phosphorylation state dependence of these $\mathrm{mAbs}$ are listed. P[ind-core] denotes an epitope on the core domain of NFP, and it is independent of the state of phosphorylation. Other notations are defined in the text.

bation with goat anti-mouse IgG coupled to agarose (HyClone) for $4 \mathrm{hr}$ to overnight. After extensive rinsing with Tris-buffered saline containing $1 \% \mathrm{NP}-40$ and $2 \mathrm{M}$ urea, bound antigen was solubilized by boiling in $2 \%$ SDS, precipitated with cold methanol, and then analyzed by PAGE.

Monoclonal antibodies. NFL and NFM subunit specific mAbs were generated using rat NFL and NFM, respectively, as the immunogens (Lee et al., 1987). SMI 31 was purchased from Sternberger-Meyer, Inc. The specificities of the mAbs used in this study are listed in Table 1.

Phosphatase treatment. Cytoskclctons wcre prepared from dissociated cultures as described in Black et al. (1986), rinsed twice with Tris buffer (50 mM Tris- $\mathrm{HCl}, \mathrm{pH} 8.0,100 \mathrm{~mm} \mathrm{NaCl}, 1 \mathrm{~mm} \mathrm{ZnSO}_{4}$ ), scraped into $100 \mu 1$ of Tris buffer, and homogenized in $1.5 \mathrm{ml}$ tubes with plastic pestles (Kontes). The homogenate was divided into 2 or more equalsized aliquots, and 1 or more were incubated with 7-8 units of $E$. coli alkaline phosphatase (Sigma) or 150 units of calf intestine alkaline phosphatase (Boeringer Mannheim) for $18 \mathrm{hr}$ at $33^{\circ} \mathrm{C}$. The reaction was terminated by the addition of SDS to $1 \%$, followed by precipitation with 8-10 vol of cold methanol. Controls for phosphatase treatment included incubation without phosphatase and incubation with phosphatase +0.2 м phosphate. Samples were assayed for NFP by PAGF. and/or immunoblotting.

Polyacrylamide gel electrophoresis. One-dimensional SDS (1D) and 2-dimensional isoelectricfocusing $\times$ SDS (2D) PAGE were performed as described previously (Black et al., 1984; Peng et al., 1985). Labeled proteins were visualized by autoradiography or fluorography (Bonner and Laskey, 1974).

\section{Results}

\section{Neurofilament proteins of cultured sympathetic neurons}

Cultured sympathetic neurons contain the NF triplet (Lee, 1985; Black et al., 1986). NFL and NFM are the principal components of NF in 1- to 2-week-old cultures, while NFH is comparatively minor. The present studies focus on NFL and NFM. NFL and NFM are readily detected in $2 \mathrm{D}$ gels of whole-cell SDS extracts and of cytoskeletons (Fig. $1 a$; see also Black et al., 1986). NFL usually focuses as a single discrete spot, although an additional slightly more basic spot is sometimes observed (Lee et al., 1987). NFM resolves into 3 distinct species, which we have designated NFM a, b, and c, in order of increasing mobility.

Visual inspection of immunoblots or 2D gels stained with Coomasie blue suggests that NFM $a$ is the most abundant NFM variant present in cultured neurons, followed by NFM $b$ and then NFM c. This impression was confirmed quantitatively in cultures labeled with ${ }^{35} \mathrm{~S}-$ methionine continuously for $3 \mathrm{~d}$. Following analysis by 2D PAGE, NFM a, b, and $c$ were excised from the gels and their cpm quantified. The ratio of ${ }^{35} \mathrm{~S}$-methionine cpm in NFM a, b, and c, normalized to NFM c, was $5.5 \pm 2.1: 2.1 \pm 0.6: 1$ (data shown are means $\pm \mathrm{SD}, n=4$ ).

Although NFM of these cultured sympathetic neurons consistently resolves into 3 major components, additional species are frequently, though variably, observed (Fig. 2). For example, by $2 \mathrm{D}$ gel electrophoresis, NFM $\mathrm{a}$ and $\mathrm{b}$ are frequently resolved 


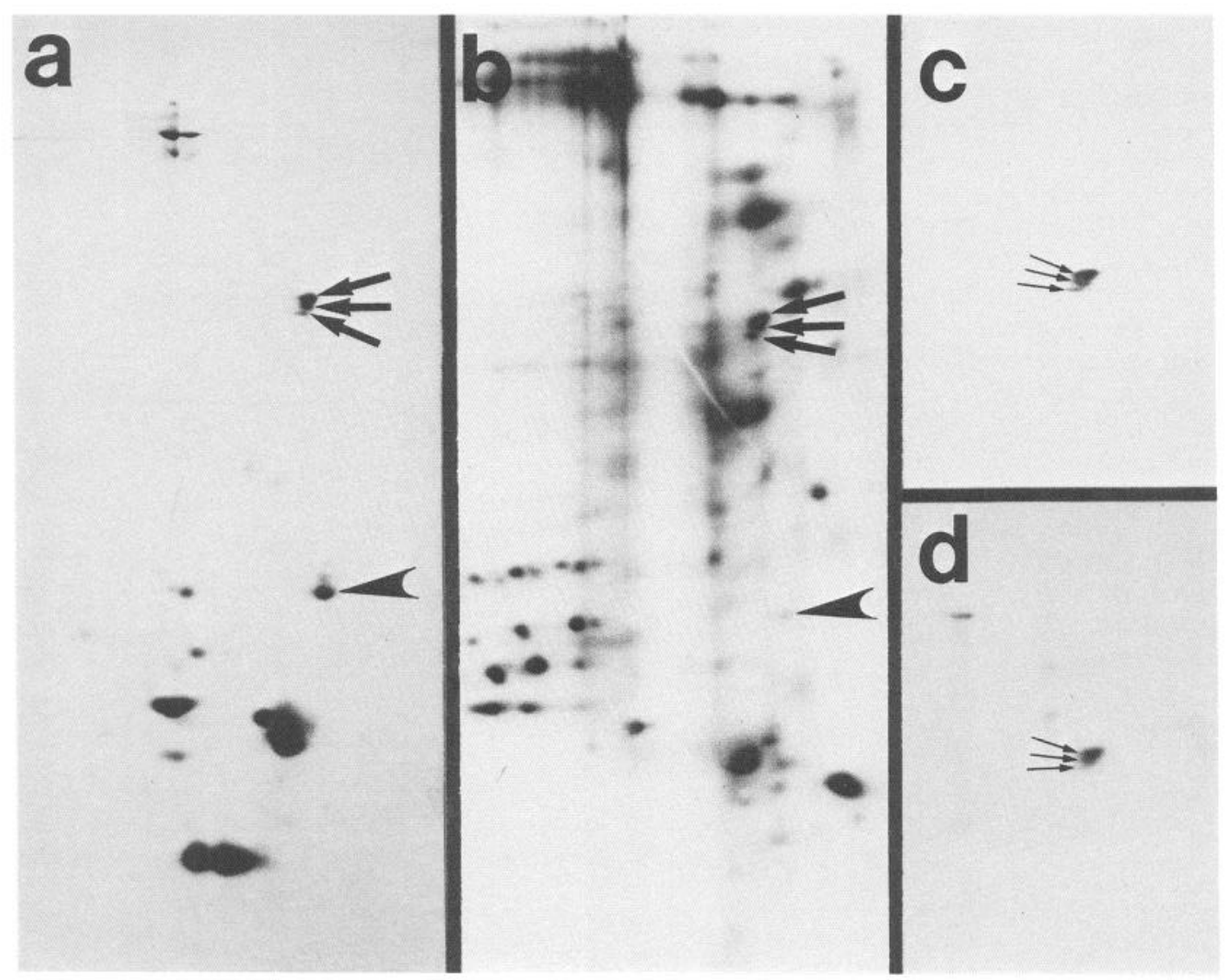

Figure 1. 2D gel analyses of neurofilament proteins in cultured sympathetic neurons. $a$, Portion of fluorograph of a $2 \mathrm{D}$ gel of a cytoskeletal preparation obtained from a culture labeled with ${ }^{35} \mathrm{~S}$-methionine for $3 \mathrm{~d}$. The arrowhead identifies NFL, while the arrows identify the 3 principal NFM species. The identification of NFL and NFM is based on Black et al. (1986). $b$, Portion of an autoradiograph of a 2D gel of a whole-cell SDS extract prepared from a culture labeled with ${ }^{32} \mathrm{P}-\mathrm{PO}_{4}$ for $2 \mathrm{hr}$. NFL and NFM are identified as in panel $a$. $c$ and $d$, Cytoskeletons from cultures incubated with ${ }^{32} \mathrm{P}_{-} \mathrm{PO}_{4}$ were assayed by $2 \mathrm{D}$ immunoblotting and autoradiography. ${ }^{32} \mathrm{P}^{-} \mathrm{PO}_{4}$-labeled cytoskeletons were resolved by $2 \mathrm{D}$ gel electrophoresis and then transferred to nitrocellulose paper. The transfer was probed with a mAb (RMO26) specific for NFM and then autoradiographed. $c$, Portion of the immunoblot containing HRP reaction product associated with NFM; the arrows identify the immunoreactive NFM spots. $d$, Autoradiographic exposure of the same portion of the transfer shown in panel $c$. Panels $c$ and $d$ are printed to the same magnification. To superimpose the autoradiogram and blot accurately, the perimeter of the blot was spotted with radioactive ink. The ink spots on the resulting autoradiogram and on the blot (not reproduced) were then used as coordinates to line up the autoradiogram on the blot. The $3{ }^{32} \mathrm{P}-\mathrm{PO}_{4}$-labeled spots indicated by the arrows in panel $d$ comigrated precisely with the NFM species revealed by immunoblotting.

into doublets (Peng et al., 1986; data not shown). The maximum number of NFM species that we have detected is 6 (Fig. $2 a$ ). These additional variants have been observed in material prepared by dissolving cells directly in buffers containing SDS and inhibitors of proteases, protein kinases, and phosphatases. We suggest that these additional variants are naturally occurring, and we attribute the variability in their detection to a combination of the subtle nature of the differences among them and the slight variability among the gradient gels required to resolve them.

\section{Phosphorylation of NFP in intact neurons}

Figure $1 b$ shows a representative autoradiograph depicting the 2D gel profile of neuronal proteins labeled with ${ }^{32} \mathrm{P}_{-}-\mathrm{PO}_{4}$. Many phosphorylated species are apparent. One relatively minor component corresponds to phosphorylated NFL; it comigrates with NFL, is quantitatively recovered in cytoskeletal preparations (data not shown), and can be immunoprecipitated with a mAb (RMS21, see Table 1) against NFL (data not shown). Three phosphorylated forms of NFM were also identified. They partitioned quantitatively with the cytoskeleton following extraction with Triton X-100 (data not shown), and they comigrated precisely with the 3 major species of NFM as illustrated by the colocalization of ${ }^{32} \mathrm{P}$-labeled NFM species with immunoreactive NFM species recognized by a mAb against a phosphate-independent site on the core domain of NFM (Fig. 1, $c, d$ ). Finally, all of these phosphorylated forms of NFM could be immunoprecipitated by a mAb against a phosphate-independent epitope (RMO26) or against a phosphorylated epitope that is present on all phosphorylated NFM species (RMO108) (Fig. 3). In other immunoprecipitation experiments, $\mathrm{RMO} 55$, a $\mathrm{P}[++]$ type $\mathrm{mAb}$, precipitates NFM a and b, whereas RMO45, a P $[+++]$ type $\mathrm{mAb}$, precipitates NFM a and, to a much lesser extent, NFM $b$ (data not shown).

\section{Effects of phosphatase treatment on NFM}

Phosphatase treatment simplifies the gel profile of NFM. When cytoskeletons from ${ }^{35} \mathrm{~S}$-methionine-labeled cultured neurons were incubated with $E$. coli or calf intestine alkaline phosphatase, only one NFM variant was detected by $2 \mathrm{D}$ gel electrophoresis, and it comigrated with NFM c (Fig. 4, $a, b$; data not shown). Similar results were also obtained when phosphatase-treated cytoskeletons were analyzed by immunoblotting, using RMO26 (Fig. $4 c$ ). The intensity of NFM $c$ in phosphatase-treated ma- 


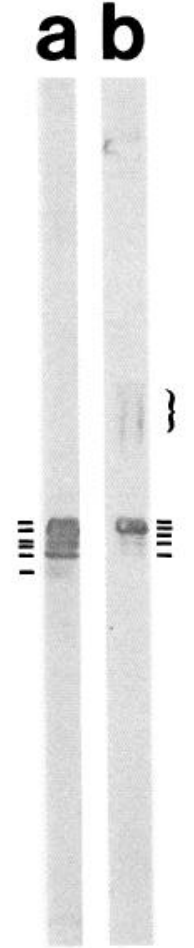

Figure 2. Immunoblot analyses of NFM. Neuronal cytoskeletons were probed for NFM by immunoblotting using RMO26 (lane $a$ ) or the SMI 31 against "highly" phosphorylated NFM + NFH (lane b). Six distinct immunoreactive bands are resolved with RMO26, while only 5 are observed with the other antibody. The band that is unique to the blot obtained with RMO26 has the mobility expected of nonphosphorylated NFM (Black et al., 1986). The immunoreactive material indicated by the bracket in lane $b$ presumably corresponds to NFH.

terial is several times greater than in control material, and this increase occurs in direct proportion to the decrease in NFM a and $b$; the total quantity of NFM did not appear to decrease following phosphatase treatment. Addition of phosphate to samples prior to addition of phosphatases blocks the conversion of NFM (Fig. 4d). We conclude that the changes in gel profile of NFM following treatment with phosphatase are due to dephosphorylation, and not proteolysis.

In other experiments, control and phosphatase-treated cytoskeletons were probed with various mAbs against phosphatedependent epitopes on NFM. For example, RMO108 gave results that were indistinguishable from those obtained with RMO26 (data not shown). Thus, the NFM variant remaining after phosphatase treatment is phosphorylated at a site (or sites) that is resistant to the phosphatases used. In this regard, our results agree with those of others that $E$. coli alkaline phosphatases do not remove all phosphate from NFP (Carden et al., 1985; Georges et al., 1986). However, it appears that certain phosphates can be readily removed from NFM. Phosphates present on NFM a and b, but not c, such as those recognized by RMO45, RMO55, and SMI 31, are very sensitive to phosphatase treatment (Fig. $4 d$; data not shown).

\section{NFL and NFM are not detectably acetylated in cultured} neurons

We have recently characterized the acetylation of cytoplasmic proteins in cultured neurons (Black and Keyser, 1987). Acetylation reduces the pI of proteins by neutralizing free amino

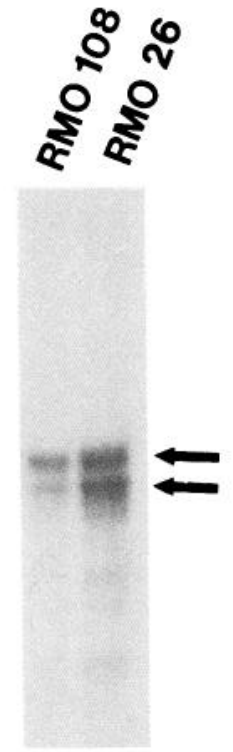

Figure 3. Immunoprecipitation of ${ }^{32} \mathrm{P}-\mathrm{PO}_{4}$-labeled NFM. Cytoskeletons from cultures labeled with ${ }^{32} \mathrm{P}-\mathrm{PO}_{4}$ for $2 \mathrm{hr}$ were assayed for labeled NFM by immunoprecipitation with RMO108 and RMO26. The upper arrow designates NFM $\mathrm{a}$ and $\mathrm{b}$, which are not well resolved from each other, and the lower arrow identifies NFM c. Note that RMO26 and RMO108 precipitate NFM a, b, and $\mathrm{c}$.

groups, and the resulting proteins migrate with slightly lower pI and higher apparent molecular weights in 2D gels. The principal acetylated protein in cultured sympathetic neurons is $\alpha$-tubulin; NFL and NFM were not detectably acetylated following incubation of cultured neurons with ${ }^{3} \mathrm{H}$-acetate for $2 \mathrm{hr}$ (data not shown).

\section{Time course of NFM phosphorylation}

We have described a variant of NFM that is apparently nonphosphorylated because it (1) is the only labeled NFM species detectable in cultures pulse-labeled with ${ }^{35} \mathrm{~S}$-methionine for 5 min and (2) is recognized by an antibody to nonphosphorylated $\mathrm{NFM}+\mathrm{NFH}$, but not by antibodies to phosphorylated forms of these proteins (Black et al., 1986). These findings are extended here by showing that NFM synthesized during a 10 min pulselabel with ${ }^{35} \mathrm{~S}$-methionine can be immunoprecipitated by phosphate-independent mAbs, such as RMO26, but not by 2 different $\mathrm{mAbs}$ to phosphate-dependent epitopes, including RMO108 (Fig. 5a). In favorable 1D gels (Figs. $2 a$ and 6) and in 2D gels (Black et al., 1986), this presumably nonphosphorylated NFM species migrates slightly faster than NFM $c$ and is present at very low levels compared with NFM a, b, or c. In other gels, this species cannot be resolved from NFM c.

To determine the approximate time interval between the synthesis and initial phosphorylation of NFM, cultures pulse-labeled with ${ }^{35} \mathrm{~S}$-methionine and chased for varying times were assayed for labeled NFM by immunoprecipitation with mAbs against a phosphate-independent epitope (RMO26) or against the phosphorylated epitope that is present on NFM a, b, and c (RMO108). As shown in Figure 5, RMO26 precipitates labeled NFM variants from cultures pulse-labeled for $10 \mathrm{~min}$ and chased for times ranging from 0 to $23 \mathrm{hr}$. In contrast, RMO108 does not precipitate labeled NFM synthesized during a $10 \mathrm{~min}$ pulselabel (Fig. 5a) but does precipitate labeled NFM from cultures pulse-labeled for $10 \mathrm{~min}$ and then chased for $60 \mathrm{~min}$ or longer 


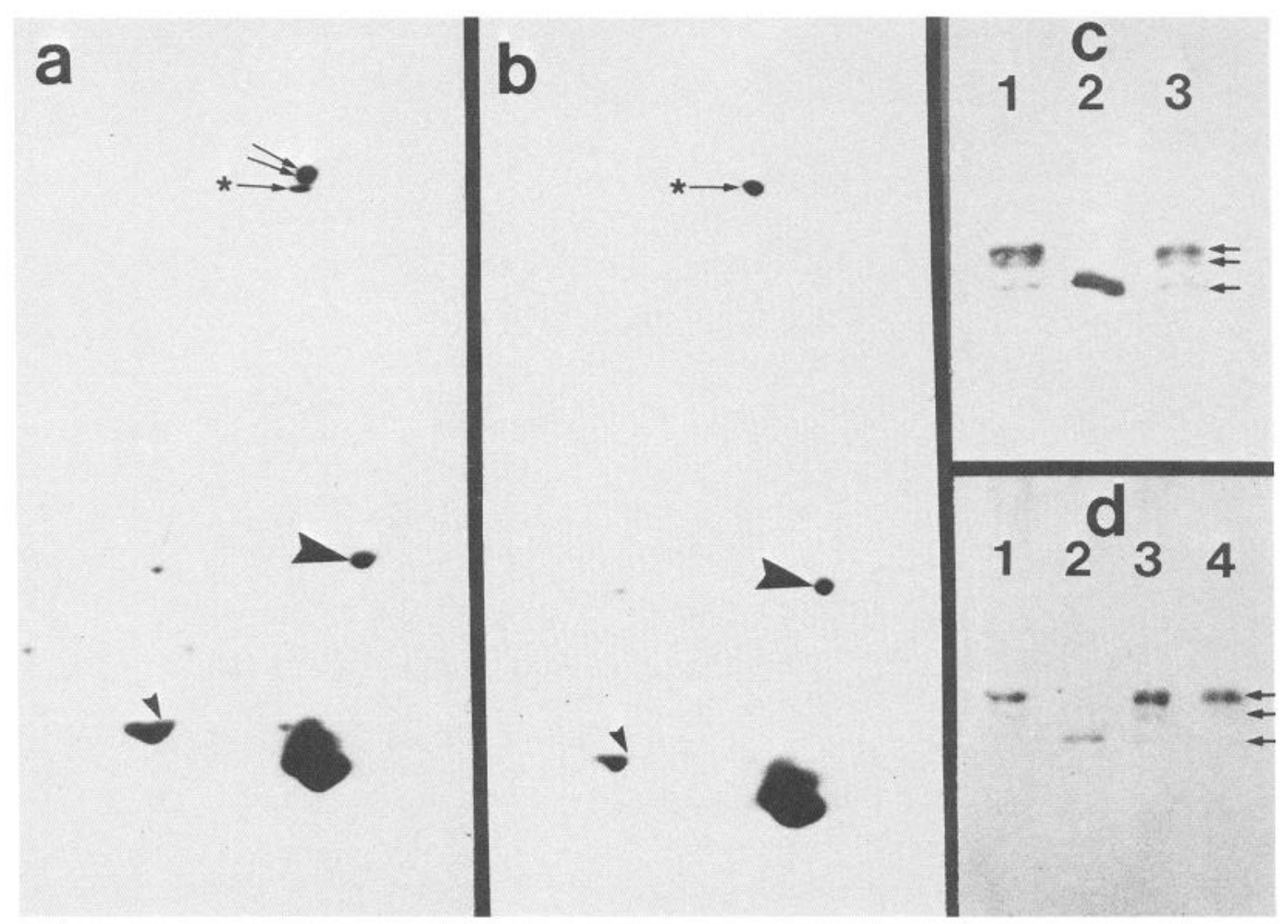

Figure 4. Effect of phosphatase treatment on the gel profile of NFM. $a$ and $b$, Cytoskeletons from cultures labeled with ${ }^{35}$ S-methionine for $3 \mathrm{~d}$ were treated with or without $E$. coli alkaline phosphatase as described in the Materials and Methods. Shown are portions of fluorograms depicting the labeled proteins in control $(a)$ or phosphatase-treated samples $(b)$. The large arrowhead identifies NFL. The arrows identify NFM a, b, and c; the arrow with asterisk indicates NFM c. The small arrowhead identifies a protein that is phosphatase-sensitive and may correspond to the intermediate filament protein recently described in PC 12 cells by Parysek and Goldman (1987). $c$ and $d$, Immunoblot analyses of NFM from freshly prepared cytoskeletons (lane 1), and cytoskeletons treated with (lane 2) or without (lane 3) phosphatase, or with phosphatase $+0.2 \mathrm{M}$ phosphate (lane 4, panel $d$ only). The blots in panel $c$ and $d$ were obtained with RMO26 and the SMI 31 , respectively.

(Fig. 5c). Thus, initial phosphorylation of NFM occurs between 10 and $60 \mathrm{~min}$ after its synthesis and generates NFM c from newly synthesized NFM. The generation of NFM $a$ and $b$ from NFM synthesized during a 10 min pulse was detected with chase times of 8 and $23 \mathrm{hr}$. Comparison of the relative labeling of NFM variants precipitated by RMO26 and RMO108 (Fig. 5, $b, c)$ suggests that newly synthesized NFM is quantitatively converted to phosphorylated forms recognized by RMO108 between 8 and $23 \mathrm{hr}$ after its synthesis.

\section{Localization of NFM variants in cultured neurons}

We have extended our previous work (Peng et al., 1986) on direct analyses of NFP in axons and in mixed preparations of cell bodies and dendrites obtained from explant cultures. In the present experiments, explant cultures were separated into CBM and proximal $(\sim 2 \mathrm{~mm})$ and $\operatorname{distal}(\sim 2 \mathrm{~mm})$ regions of the axonal halo. The resulting preparations were probed for NFM variants by immunoblotting, using RMO26, RMO108, RMO55, and RMO45. RMO26 reveals 4 distinct species of NFM in the CBM (Fig. 6, lane 1); three correspond to NFM a, b, and c. The remaining species migrates slightly faster than NFM c, and it is tentatively identified as nonphosphorylated NFM. In contrast, only NFM $a$ and $b$ were detected in both proximal and distal regions of the axonal halo (Fig. 6, lanes 2, 3). The relative abundances of these 2 variants are comparable in the proximal and distal regions of the axonal halo. Comparable observations were also obtained with explant cultures of dorsal root ganglion neurons (Fig. 6, lanes 4, 5). Similar patterns of NFM a, b, and c distribution between CBM and axons were also observed with RMO108, RMO55, and RMO45 (Fig. 6, lanes 6, 7, and data not shown). We have also consistently noted very subtle differences between NFM $a$ and $b$ of CBM and axons. In particular, careful inspection of the blots of Figure 6 suggests that NFM a and $\mathrm{b}$ of axons do not precisely comigrate with NFM $\mathrm{a}$ and $\mathrm{b}$ of the CBM (see also Fig. 5 of Peng et al., 1986).

\section{Phosphorylation of NFP in axons}

We have used explant cultures to determine whether NFP are phosphorylated locally in axons. CBM and pure axon preparations from ${ }^{32} \mathrm{P}_{-} \mathrm{PO}_{4}$-labeled explant cultures were assayed for phosphorylated NFP by PAGE and autoradiography. Many proteins can be phosphorylated in the CBM and axons, and many differences in the pattern of phosphorylated proteins generated in these regions are apparent. (A more detailed characterization of the pattern of phosphorylated proteins generated in these regions will be presented in a separate manuscript.) Both NFL and NFM were phosphorylated in CBM and axons (Fig. 7, $a$, $b$ ), and as expected, the phosphorylated NFP of axons partition with the detergent-insoluble cytoskeleton (Fig. 7c). The identification of ${ }^{32} \mathrm{P}$-phosphate-labeled NFM in axons was confirmed by immunoprecipitation using RMO26 (data not shown). Identical results were obtained when intact explant cultures were 


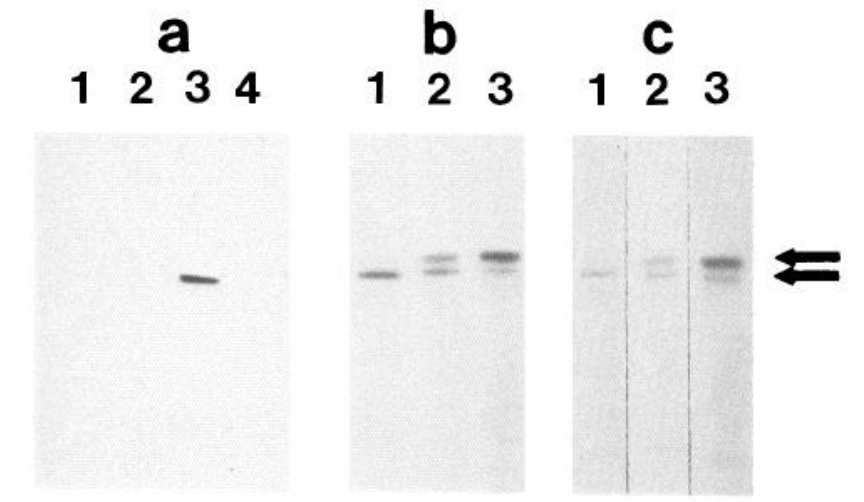

Figure 5. Immunoprecipitation of ${ }^{35} \mathrm{~S}$-methionine-labeled NFM. $a$, An SDS extract of cultures labeled for $10 \mathrm{~min}$ was divided into 4 equal aliquots, and each was assayed for NFM by immunoprecipitation. Shown is a portion of a fluorograph depicting immunoprecipitates obtained with RMO55 (lane 1), RMO108 (lane 2), RMO26 (lane 3), and no first antibody (lane 4). $b$ and $c$, Labeled NFM species from cultures pulselabeled for $15 \mathrm{~min}$ and then chased for $1 \mathrm{hr}$ (lane 1), $8 \mathrm{hr}$ (lane 2), or $23 \mathrm{hr}$ (lane 3 ) obtained by immunoprecipitation with RMO26 (b) or RMOl08 $(c)$. NFM a and b are not well resolved from each other and are indicated by the upper arrow. The lower arrow indicates NFM c. In all of these immunoprecipitation analyses, sufficient antibody was used for quantitative precipitation of the respective antigens. This was established by probing the nonprecipitated material for NFM by immunoblotting; in all cases, no NFM was detected in the nonprecipitated material. Thus, in panel $a$, the newly synthesized NFM is precipitated by RMO26, which recognizes a phosphate-independent epitope on NFM, but not by RMO108 or RMO55, both of which recognize phosphatedependent epitopes (see Table 1). We conclude that the phosphorylation events that generate the epitopes recognized by RMO108 and RMO55 do not occur within the first $10 \mathrm{~min}$ after NFM is synthesized. However, the RMO108 epitope is generated during the first hour after synthesis (panel $c$ ).

labeled and then separated into CBM and axons or when cultures were first separated into CBM and axons prior to labeling. Phosphorylated NFL generated in the CBM was indistinguishable from that generated in the axonal halos with respect to mobility in 2D gels. We consistently observed that NFL in CBM was more heavily labeled than in axons. This may in part reflect differences in the amount of NFL in the CBM and axonal halo (see Peng et al., 1986).

The pattern of phosphorylated NFM variants generated in the CBM was distinct from that generated in the axonal halo. NFM a, b, and c were all phosphorylated in the CBM, and with the labeling conditions used, NFM $\mathrm{c}$ was the most prominent phosphorylated variant generated in the CBM, followed by NFM $\mathrm{b}$, and then NFM a. This is in marked contrast to the phosphorylation pattern observed in whole neurons, in which NFM $\mathrm{a}$ is the most prominent phosphorylated variant generated, followed by NFM $b$ and then NFM c (see Fig. 1).

The pattern of phosphorylated NFM variants generated in the axonal halo was markedly different from that observed in $\mathrm{CBM}$ and in whole cells. Only NFM a and b were detectably phosphorylated in the axons, and of these, NFM a was much more heavily labeled than NFM b. Comparable observations were also obtained when the distal $1 / 3-1 / 2$ of the axonal halo was analyzed.

Phosphorylation of both NFL and NFM can also be demonstrated in axons of peripheral nerve. We observed that NFM $a$ and $b$ were phosphorylated in isolated pieces of sciatic nerve

\section{7}

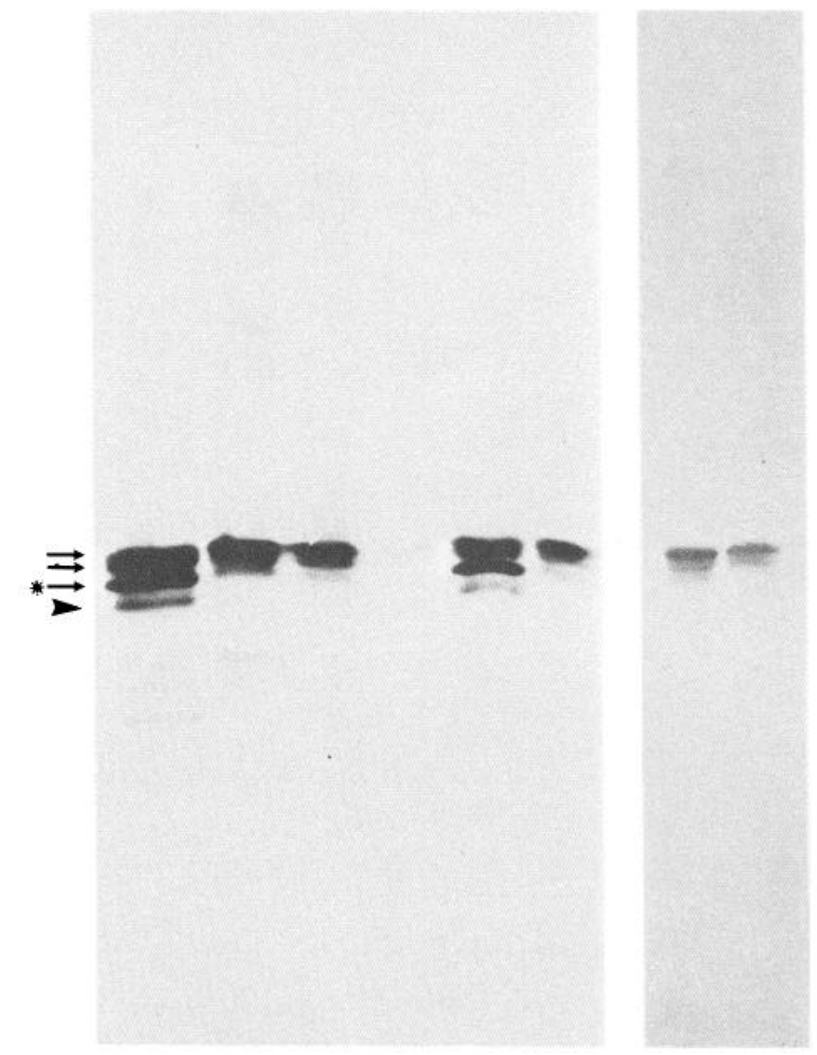

Figure 6. Immunoblot analyses of NFM in CBM and axonal halos of explant cultures of sympathetic and dorsal root ganglion neurons. Lanes $1-3$, NFM species in CBM, proximal $2 \mathrm{~mm}$ of the axonal halo and distal $2 \mathrm{~mm}$ of the axonal halo, respectively, from an explant culture of sympathetic neurons. Lanes 4 and 5, NFM species in the CBM and axonal halo from an explant culture of dorsal root ganglion neurons. Lanes 6 and 7, NFM species in CBM and axons, respectively, of sympathetic neurons as revealed by RMO55. The preparations were resolved on gels consisting of a 4-8\% gradient of acrylamide. The arrowhead identifies nonphosphorylated NFM, while the arrows identify the 3 principal phosphorylated variants of NFM; the arrow with asterisk identifies NFM c.

from young (14-d-old) and adult (120-d-old) rats incubated with ${ }^{32} \mathrm{P}^{-} \mathrm{PO}_{4}$ (Fig. 8).

\section{Discussion}

Our studies reveal extensive heterogeneity in NFM of cultured neurons. Typically, 3 variants of the NFM are resolved by gel electrophoresis, although, as many as 6 distinct species can be detected (see Figs. 1, 2, 5). Heterogeneity has also been observed in NFM of axons of mature neurons (Nixon et al., 1982). This heterogeneity appears to be naturally occurring (see Results) and, as argued below, is due almost entirely to phosphorylation.

We know that NFM from adult nervous tissue is multiply phosphorylated in situ (Jones and Williams, 1982; Wong et al., 1984; Carden et al., 1985; Georges et al., 1986; Ksiezak-Reding and Yen, 1987) and that NFM of cultured neurons is also phosphorylated in situ. Theoretically, NFM variants that differ in phosphate content should have slightly different mobilities in 1D and 2D gels. Thus, one interpretation of the heterogeneity of NFM seen by gel electrophoresis is that cultured neurons 


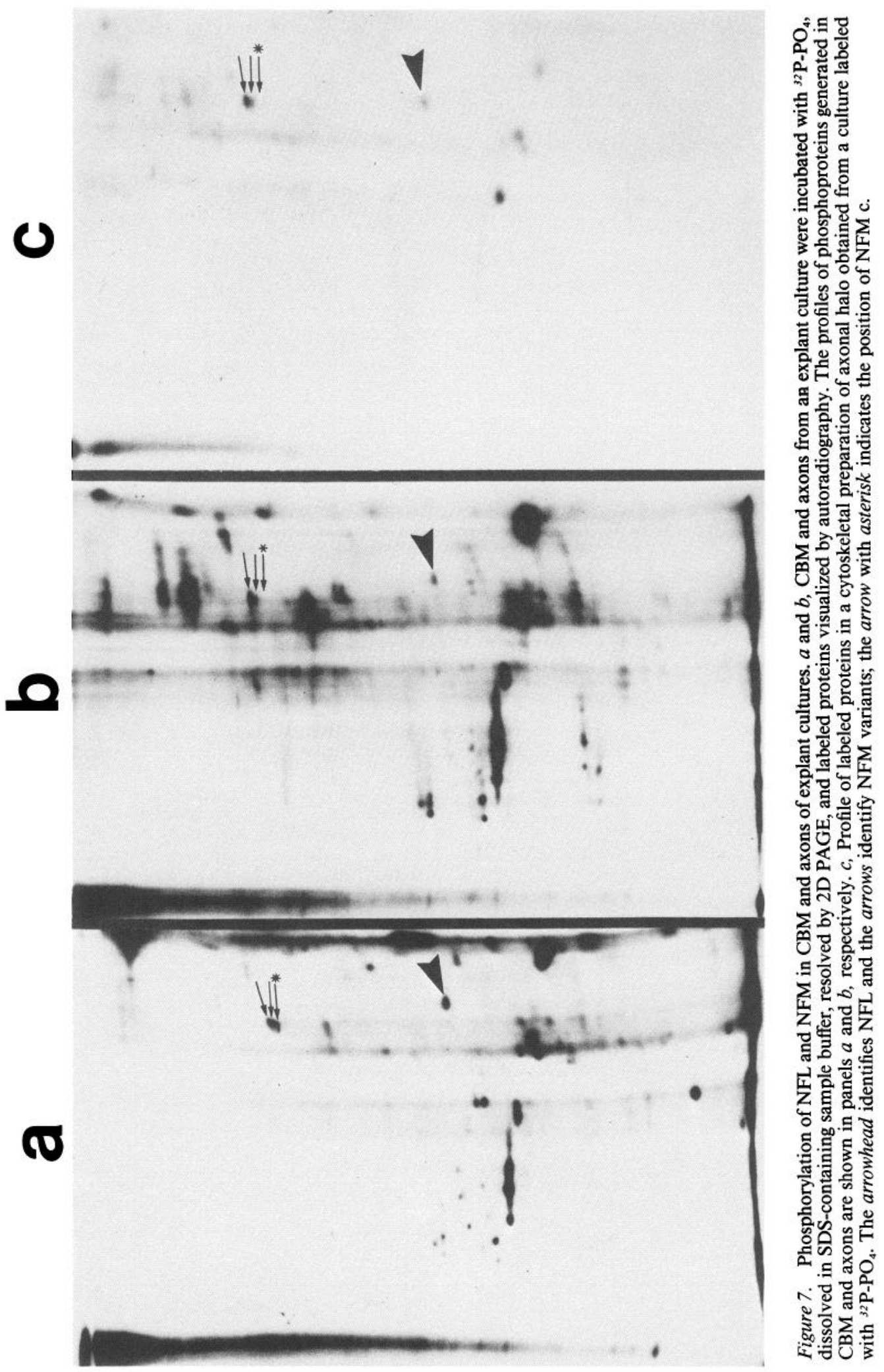


normally contain a variety of NFM species that differ in phosphate content. This interpretation is fully supported by the observations that (1) all but one of the NFM variants can incorporate ${ }^{32} \mathrm{P}-\mathrm{PO}_{4}$ in situ (Figs. 1, 7), (2) the heterogeneity of NFM can be reduced following treatment with phosphatases (Fig. 4), (3) NFM is not known to undergo any other charge modifying posttranslational modification such as acetylation, and (4) phosphorylated NFM variants can be recognized by several welldefined mAbs such as RMO108, RMO55, RMO45, and SMI 31 , which recognized different phosphorylation states and also distinct phosphate-dependent epitopes on NFM(Table 1). Thus, we can define at least 4 different phosphorylated epitopes on NFM of cultured sympathetic neurons, suggesting at least 4 distinct phosphorylation sites on the protein in cultured neurons. We have observed 6 electrophoretically distinct species of NFM, of which 5 are phosphorylated. If these 5 phosphorylated species differ solely in phosphate content, then at least one phosphate site in addition to those defined by the above-described mAbs is present on NFM of cultured neurons. Variations in the phosphorylation of these sites may thus account for the heterogeneity of NFM observed in neurons.

The above considerations suggest at least 5 phosphorylation sites on rat NFM. The exact location and amino acid reference of these phosphorylation sites are presently unknown. Recently, the genes for both human and rat NFM have been cloned (Meyer et al., 1987; Napolitano et al., 1987, respectively). Upon examination of the gene-derived amino acid sequences of human and rat NFM, potential phosphorylation sites can be identified. In human, a 13 amino acid peptide with the sequence KSPVPKSPVEEKG was found to be repeated in tandem 6 times. We have shown, using a combination of synthetic peptides with or without chemical phosphorylation and $\mathrm{mAbs}$, that this repeat domain represents a multiphosphorylation site in human NFM (Lee et al., 1988). Thus, the repeat sequence KSP is the phosphorylation site in human NFM. In rat, $5 \mathrm{KSPs}$ are present, although these KSPs are not found in tandem (Napolitano et al., 1987). If these 5 KSPs indeed represent the phosphorylation sites in rat NFM, this may explain the presence of 5 distinct phosphorylation variants observed in our experiments. Direct identification of the phosphorylation sites on NFM of cultured neurons is required to substantiate this possibility.

We have extended our earlier studies (Peng et al., 1986) on the composition of NFM in cell bodies, dendrites plus proximal $(\sim 100 \mu \mathrm{m})$ axons versus pure distal axon preparations of cultured neurons. These analyses are unique in that they provide detailed information on the specific NFM variants present in particular regions of neurons. This information strongly complements that revealed by immunostaining analyses; these provide information on the localization of specific NFM epitopes, which are generally present on more than one NFM variant (see Figs. 5 and 6 for examples).

The results of our immunoblotting analyses with explant cultures taken together with those of immunostaining studies indicate that non- and poorly phosphorylated NFM variants are present in the cell body and proximal $100 \mu \mathrm{m}$ or so of the axon but are not detectable more distally in the axon, while more highly phosphorylated NFM variants are greatly enriched in axons compared with the cell body (Fig. 6; see also Peng et al., 1986; Lee et al., 1987). These observations indicate that considerable phosphorylation of NFP normally occurs in the proximal part of the axon, and in the cell body as well (see below).

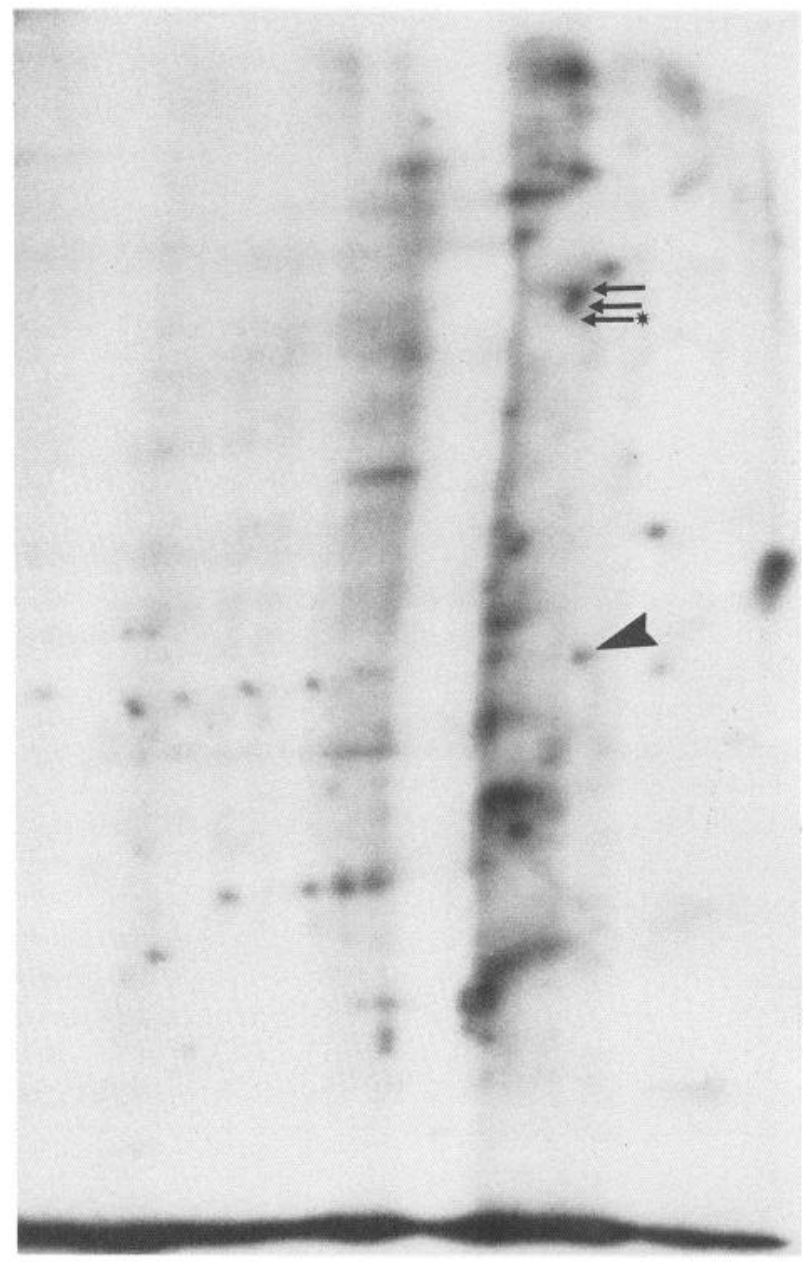

Figure 8. Phosphorylation of NFL and NFM in isolated pieces of nerve. An $\sim 0.5 \mathrm{~cm}$ piece of sciatic nerve was incubated with ${ }^{32} \mathrm{P}_{-} \mathrm{PO}_{4}$ for $60 \mathrm{~min}$, dissolved in sample buffer and then analyzed by $2 \mathrm{D}$ PAGE and autoradiography. A portion of a representative autoradiogram is shown, with the arrowhead and arrows indicating labeled spots that comigrate with NFL and NFM, respectively.

It is not known, however, how the phosphorylation state of NFP is regulated more distally in the axon. Resolving this issue is essential for a full understanding of the functional significance of NFP phosphorylation. For example, it is possible that NFP become maximally phosphorylated in the very proximal region of the axon, and the resulting phosphates do not turn over until the proteins themselves turn over. In this view, phosphorylation does not represent a means of dynamically altering the proteins because they are maximally and permanently modified in the initial region of the axon. An alternative view is that phosphorylation represents a means of dynamically altering NFP and NF in response to changes in the internal and/or external environment of the axon. If this is correct, then NFP phosphorylation should occur throughout the axon. It is also possible that both of the above-mentioned views are correct in that some phosphates may be stably incorporated into NFP within the cell body and/or proximal axonal domains, while other phosphates are cyclically added and removed at more distal sites within the axon. Nixon and coworkers (Nixon et al., 1982, 1987; Nixon and Lewis, 1986) have presented evidence consistent with this last possibility. They showed that some of the phosphate in- 
corporated into NFP following intraocular injection of ${ }^{32} \mathrm{P}-\mathrm{P}_{\mathrm{i}}$ persists for several days. They also observed subtle changes in the pI of NFP during their transport within the optic axons, suggesting that they are modified locally within the axon. The nature of this modification was not demonstrated, although it seems likely that it involved phosphorylation. 'lo evaluate further the modification of NFP locally within axons, we have used explant cultures to study phosphorylation of NFP in purc axonal preparations.

In these explant cultures, NFL and NFM in the CBM and axonal halo become labeled following short-term incubation with ${ }^{32} \mathrm{P}_{-} \mathrm{PO}_{4}$ (Fig. 7). Phosphorylation of NFL and NFM occurred in the proximal $2 \mathrm{~mm}$ of the axonal halo as well as the distal $2 \mathrm{~mm}$, and there were no obvious differences in the profile of labeled NFP generated in these axonal regions. We consistently observed differences between the profile of labeled NFP seen in CBM versus axonal halo. For example, the degree of labeling of NFL was consistently greater in the CBM than the axons (see Fig. 7 and Results for further discussion).

Also, the pattern of labeled NFM variants generated in the $\mathrm{CBM}$ is very different from that generated in the axonal halo. The basis for this is that newly synthesized NFM undergoes progressive phosphorylation that begins within the first hour of its synthesis and continues for hours to days (Fig. 5; see also Bennett and DiLullo, 1985; Black et al., 1986). Among the earliest events in the phosphorylation sequence of NFM are those that generate the phosphate-dependent epitope defined by RMO108; they occur between $10 \mathrm{~min}$ and $1 \mathrm{hr}$ after the synthesis of NFM (Fig. 5) and result in the generation of NFM $c$ from newly synthesized NFM. The time interval between the synthesis of NFM and its conversion to NFM $c$ is very short relative to that of the synthesis of NFM and its transport into axons, which is on the order of 16-24 hr (Black, unpublished observations). This suggests that the phosphorylation events involved in generating NFM $c$ are completed within the cell body, an interpretation fully supported by the observation that the incorporation of phosphate into NFM c occurs prominently and only in the CBM of explant cultures (Fig. 7). The next series of phosphorylation events converts NFM $c$ to NFM b (Fig. 5; see also Black et al., 1986). [Recall that NFM b is hetergeneous (Fig. 3).] Some of these events are also largely completed in the cell body and/or proximal axonal compartments because NFM $\mathrm{c}$ is prominent in these compartments but is present at only trace levels more distally in the axons (Fig. 6). On the other hand, the phosphorylation events that generate NFM a occur later in the sequence (Fig. 5; see also Black et al., 1986) and occur prominently in the axons but only to limited extent in the cell body and/or very proximal axonal compartments (Figs. 6 , and 7). The regional differences in phosphorylation of NFM may reflect the existence of a rigid sequence to the phosphorylation of newly synthesized NFM which is played out slowly relative to the rate at which this protein is transported from the cell body into the axons. The slow phosphorylation of NFM may involve multiple kinases, with some active in the cell body plus proximal axonal compartments, while others are active more distally in the axons.

The important point from the present data is that NFL and NFM can be phosphorylated in neuronal cell bodies and distal as well as proximal locations within axons. The latter observation is not merely a property of immature neurons or neurons grown in culture because we observed phosphorylation of NFL and NFM in isolated pieces of nerve obtained from adults rats
(Fig. 8; see also Nixon et al., 1987). The fact that NFP can be phosphorylated at distal sites within axons during short-term incubations with ${ }^{32} \mathrm{P}-\mathrm{PO}_{4}$ (Fig. 7) and can also be dephosphorylated within the axon (Nixon et al., 1982; Nixon and Lewis, 1986) indicates that phosphorylation and dephosphorylation of these proteins occur cyclically in axons. This in turn suggests that phosphorylation potentially represents a means of dynamically regulating aspects of NF structure and function.

\section{References}

Black, M. M., and P. Keyser (1987) Acetylation of $\alpha$-tubulin in cultured neurons and the induction of $\alpha$-tubulin acetylation in PC 12 cells by treatment with nerve growth factor. J. Neurosci. 7: 18331842.

Black, M. M., M. Cochran, and I. T. Kurdyla (1984) Solubility properties of neuronal tubulin: Evidence for labile and stable microtubules. Brain Res. 295: 255-263.

Black, M. M., P. Keyser, and E. Sobel (1986) Interval between the synthesis and assembly of cytoskeletal proteins in cultured neurons. J. Neurosci. 6: 1004-1012.

Bennett, G. S., and C. DiLullo (1985) Slow posttranslational modification of a neurofilament protein. J. Cell Biol. 100: 1799-1804.

Bonner, W. M., and R. A. Laskey (1974) A film detection method for tritium-labeled proteins and nucleic acids in polyacrylamide gels. Eur. J. Biochem. 46: 83-88.

Carden, M. J., W. W. Schlaepfer, and V. M.-Y. Lee (1985) The structure, biochemical properties and immunogenicity of neurofilament peripheral regions are determined by phosphorylation state. J. Biol. Chem. 260: 9805-9817.

Carden, M. J., J. Q. Trojanowski, W. W. Schlaepfer, and V. M.-Y. Lee (1987) Two-stage expression of neurofilament polypeptides during rat neurogenesis with early establishment of adult phosphorylation patterns. J. Neurosci. 7: 3489-3504.

Geisler, N., E. Kaufman, S. Fischer, U. Plessmann, and K. Weber (1983) Neurofilament architecture combines structural principles of intermediate filaments with carboxy-terminal extensions increasing in size between triplet proteins. EMBO J. 2: 1295-1302.

Georges, E., S. Lefebvre, and W. E. Mushynski (1986) Dephosphorylation of neurofilaments by exogenous phosphatases has no effect on reassembly of subunits. J. Neurochem. 47: 477-483.

Hoffman, P. N., and R. J. Lasek (1975) The slow component of transport: Identification of major structural polypeptides of the axon and their generality among mammalian neurons. J. Cell Biol. 66: 351366.

Jones, S. M., and R. C. Williams (1982) Phosphate content of mammalian neurofilament polypeptides. J. Biol. Chem. 257: 9902-9905.

Julien, J.-P., and W. E. Mushynski (1982) Multiple phosphorylation sites in mammalian neurofilament polypeptides. J. Biol. Chem. 257: 10467-10470.

Julien, J.-P., and W. E. Mushynski (1983) The distribution of phosphorylation sites among identified proteolytic fragments of mammalian neurofilaments. J. Biol. Chem. 258: 4019-4025.

Ksiezak-Reding, H., and S.-H. Yen (1987) Phosphatase and carbocyanine dye binding define different types of phosphate groups in mammalian neurofilaments. J. Neurosci. 7: 3474-3488.

Lee, V. M.-Y. (1985) Neurofilament protein abnormalities in PC12 cells: Comparison with neurofilament protein of normal cultured rat sympathetic neurons. J. Neurosci. 5: 3039-3046.

Lee, V. M.-Y., M. J. Carden, and J. Q. Trojanowski (1986) Novel monoclonal antibodies provide evidence for the in situ existence of a nonphosphorylated form of the largest neurofilament subunit. J. Neurosci. 6: 850-858.

Lee, V. M.-Y., M. J. Carden, W. W. Schlaepfer, and J. Q. Trojanowski (1987) Monoclonal antibodies distinguish several differentially phosphorylated states of the two largest rat neurofilament subunits (NF-H and NF-M) and demonstrate their existence in the normal nervous system of adult rats. J. Neurosci. 7: 3474-3488.

Lee, V. M.-Y., L. Otvos, Jr., M. J. Carden, M. Hollosi, B. Dietzchold, and R. A. Lazzarini (1988) Identification of the major multi-phosphorylation site in mammalian neurofilaments. Proc. Natl. Acad. Sci. USA 85: 1998-2002.

Liem, R. K. H., S.-H. Yen, G. D. Salomon, and M. L. Shelanski (1978) Intermediate filaments in nervous tissue. J. Cell Biol. 79: 637-645. 
Meyer, M. W., R. A. Lazzarini, V. M.-Y. Lee, W. W. Schlaepfer, and D. L. Nelson (1987) The human mid-size neurofilament subunit: A repeated protein sequence and the relationship of its gene to the intermediate filament gene family. EMBO J. 6: 1617-1626.

Napolitano, E. W., S. S. M. Chin, D. R. Colman, and R. K. H. Liem (1987) Complete amino acid sequence and in vitro expression of rat NF-M, the middle molecular weight neurofilament protein. J. Neurosci. 7: 2590-2599.

Nixon, R. A., and S. E. Lewis (1986) Differential turnover of phosphate groups on neurofilament subunits during axonal transport. J. Biol. Chem. 261: 16278-16301.

Nixon, R. A., B. A. Brown, and C. A. Morotta (1982) Posttranslational modification of a neurofilament protein during axoplasmic transport: Implications for regional specialization of CNS axons. J. Cell Biol. 94: $150-158$.

Nixon, R. A., S. E. Lewis, and C. A. Morotta (1987) Posttranslational modification of neurofilament proteins by phosphate during axoplasmic transport in retinal ganglion cell neurons. J. Neurosci. 7: $1145-1158$

Oblinger, M. M. (1987) Characterization of posttranslational processing of the mammalian high molecular weight NF protein in vivo. J. Neurosci. 7: 2510-2521.
Parysek, L. M., and R. D. Goldman (1987) Characterization of intermediate filaments in PC12 cells. J. Neurosci. 7: 792-806.

Peng, I., L. I. Binder, and M. M. Black (1985) Cultured sympathetic neurons contain a variety of microtubule-associated proteins. Brain Res. 361: 200-211.

Peng, I., L. I. Binder, and M. M. Black (1986) Biochemical and immunological analyses of cytoskeletal domains of neurons. J. Cell Biol. 99: 2157-2164.

Peters, A., S. L. Palay, and H. deWebster (1976) The Fine Structure of the Nervous System: The Neurons and Supporting Cells, Saunders, Philadelphia.

Schlaepfer, W. W., and L. A. Freeman (1978) Neurofilament proteins of rat peripheral nerve and spinal cord. J. Cell Biol. 78: 653-662.

Sternberger, L. A., and N. H. Sternberger (1983) Monoclonal antibodies distinguish phosphorylated and nonphosphorylated forms of neurofilaments in situ. Proc. Natl. Acad. Sci. USA 80: 6126-6130.

Wong, J., S. B. Hutchison, and R. H. K. Liem (1984) An isoelectric variant of the 150,000-dalton neurofilament polypeptide. Evidence that phosphorylation affects its association with the filament. J. Biol. Chem. 259: 10867-10874. 